\title{
Photoinduced Paramagnetic Centers in Nanocomposites Formed by Titanium Dioxide and Myristic Acid
}

\author{
Claudio José Magon*, Harold Lozano Zarto, José Pedro Donoso, Hellmut Eckert
} Instituto de Física de São Carlos, Universidade de São Paulo, P.O. Box 369, 13560-970 São Carlos, SP, Brasil

Sindy Devis

Instituto de Investigación Interdisciplinar en Ciencias Biomédicas. Facultad de Ciencias de la Salud. Universidad SEK, Santiago, Chile

\section{Eglantina Benavente}

Departamento de Química, Facultad de Ciencias Naturales, Matemática y Medio Ambiente Universidad Tecnológica Metropolitana, Santiago, Chile

Programa Institucional de Fomento a la Investigación, Desarrollo e Innovación (PIDi), Universidad Tecnológica Metropolitana, P.O. Box 9845, Santiago, Chile

Roberto Villarroel

Instituto de Física, Pontificia Universidad Católica de Chile, Santiago, Chile

\section{Guillermo González*}

Departamento de Química, Facultad de Ciencias, Universidad de Chile, Santiago, Chile

\section{SUPPORTING MATERIALS SECTION}




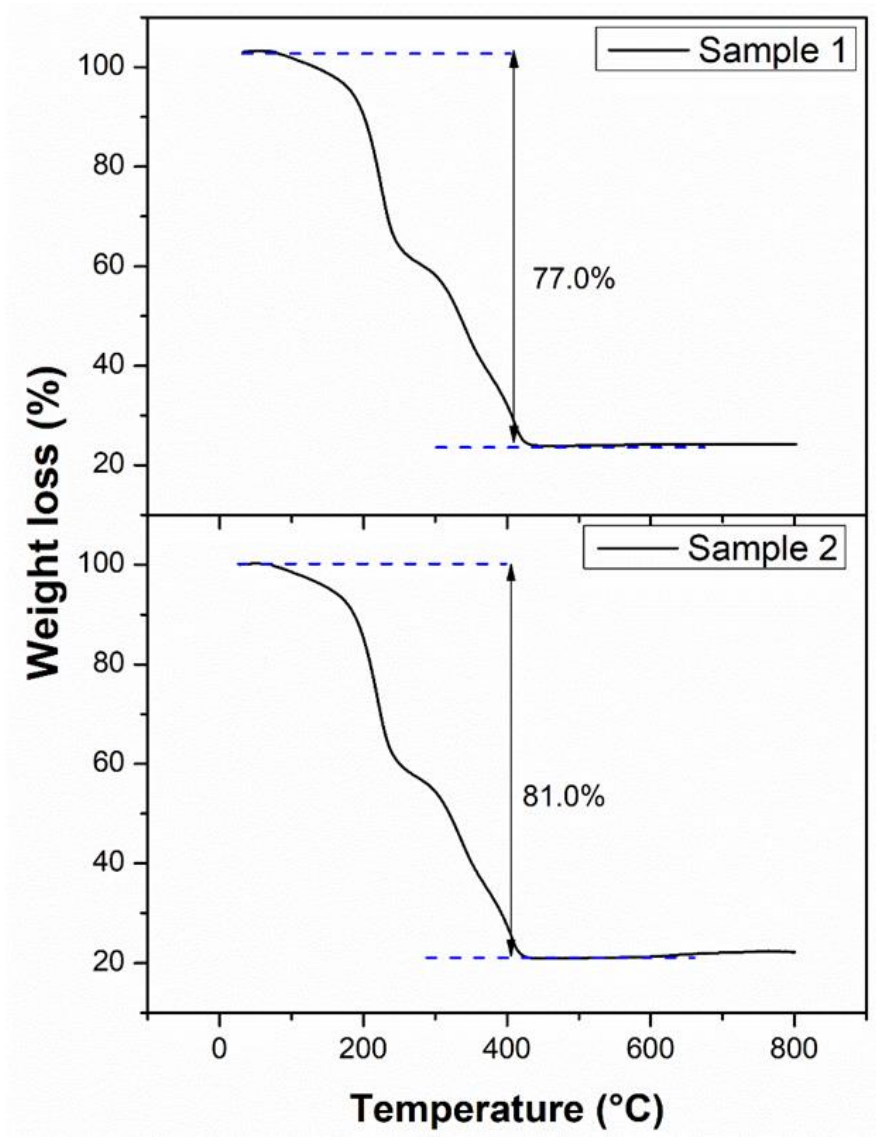

Figure S1. Thermogravimetric analysis for samples 1 and 2. Composition of the products determined by elemental analysis (SISONS ES-1108) and thermogravimetric analyses (TG/DSC 1100 SF Mettler Toledo). Sample 1: $\mathrm{TiO}_{2}\left(\mathrm{C}_{14} \mathrm{H}_{28} \mathrm{O}_{2}\right)_{1.17} \bullet\left(\mathrm{H}_{2} \mathrm{O}\right)_{0.10}$ : Calcd. C, 56.41; H, 9.459. Found C, 56.62; H, 9.530; $\mathrm{TiO}_{2} ; 22.9$ (77,1\% TG). Sample 2: $\mathrm{TiO}_{2}\left(\mathrm{C}_{14} \mathrm{H}_{28} \mathrm{O}_{2}\right)_{1.50} \bullet\left(\mathrm{H}_{2} \mathrm{O}\right)_{0.10}$ : Calcd. C, 59.48; H, 9.960. Found C, 60.10; H, 10.09; $\mathrm{TiO}_{2}$; $18.9(81,1 \% \mathrm{TG})$. 


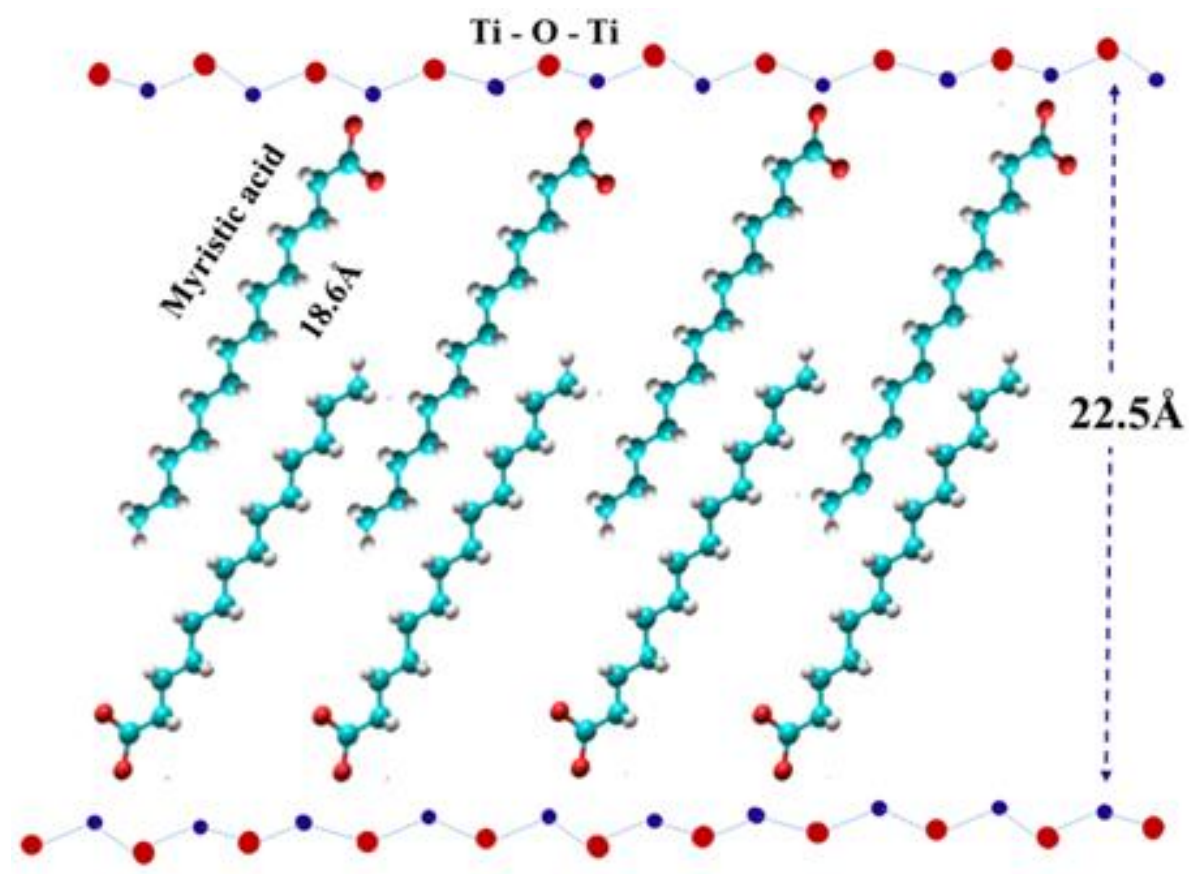

Figure S2. Schematic illustration of nanostructured LHMATi. 

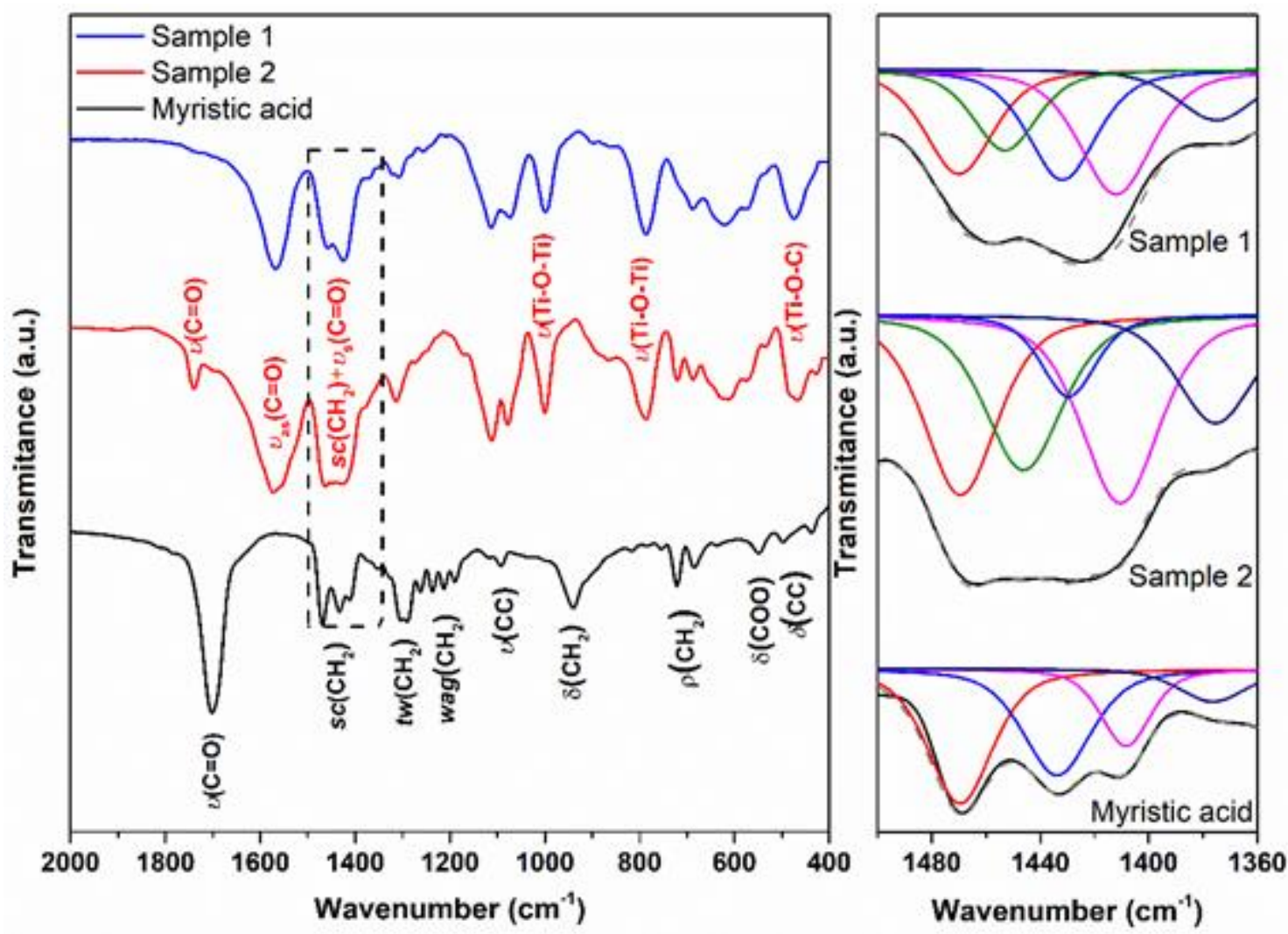

Figure S3. FTIR spectra of MA and of samples 1 and 2. Deconvolution of the $\operatorname{sc}\left(\mathrm{CH}_{2}\right)$ and $s c\left(\mathrm{CH}_{2}\right)+v_{\mathrm{s}}(\mathrm{C}=\mathrm{O})$ bands of both MA and LHMATi samples (dashed line marked area), respectively. [J.R.S. Miranda, A.J.R. de Castro, J.G. da Silva Filho, P.T.C. Freire, G.S. Pinheiro, G.D. Saraiva, F.F. de Sousa, Phase transformation in the C form of myristic-acid crystals and DFT calculations, Spectrochim. Acta Part A 2019, 208, 97108]. 


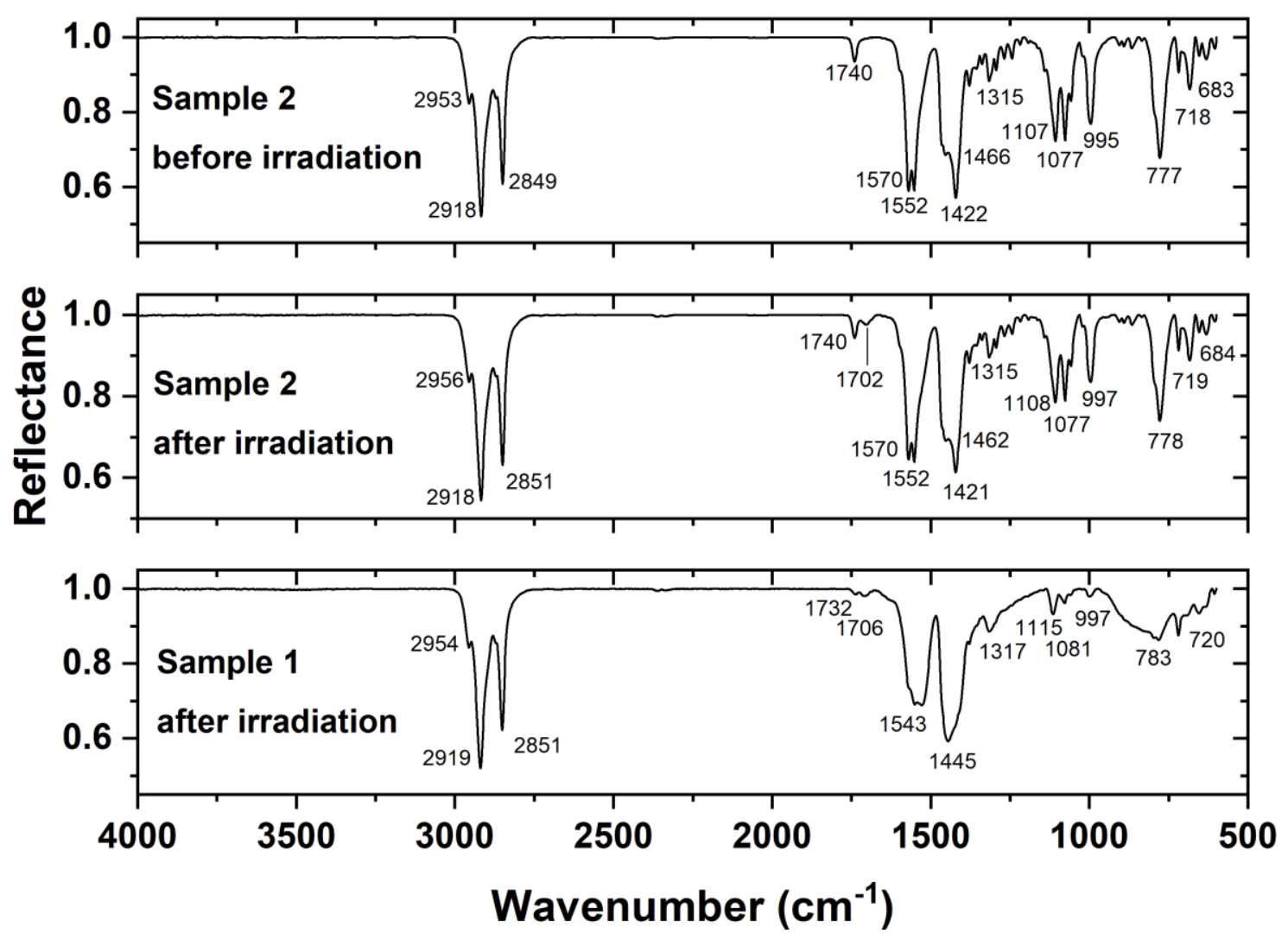

Figure S4a. FTIR results obtained on the samples under study. illustrating the differences between sample 1 and sample 2 (before and after UV exposure). The wavenumbers of the main spectroscopic features are indicated. Data recorded on a Bruker 27 FT-IR spectrometer. 


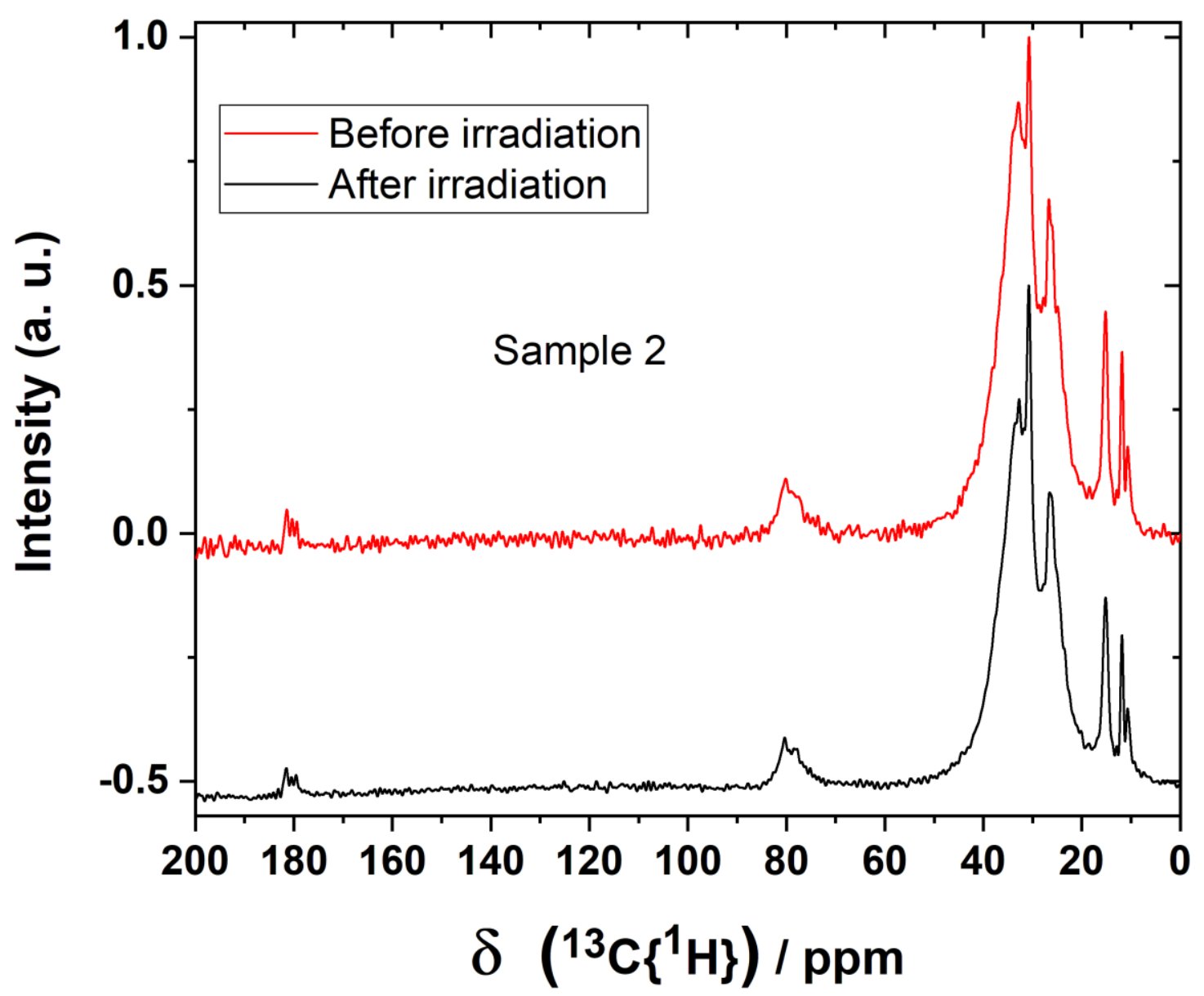

Figure S4b. ${ }^{13} \mathrm{C}$ CPMAS NMR spectra of sample 2 before irradiation and $24 \mathrm{~h}$ after irradiation for $1 \mathrm{~h}$ at $365 \mathrm{~nm}$. 

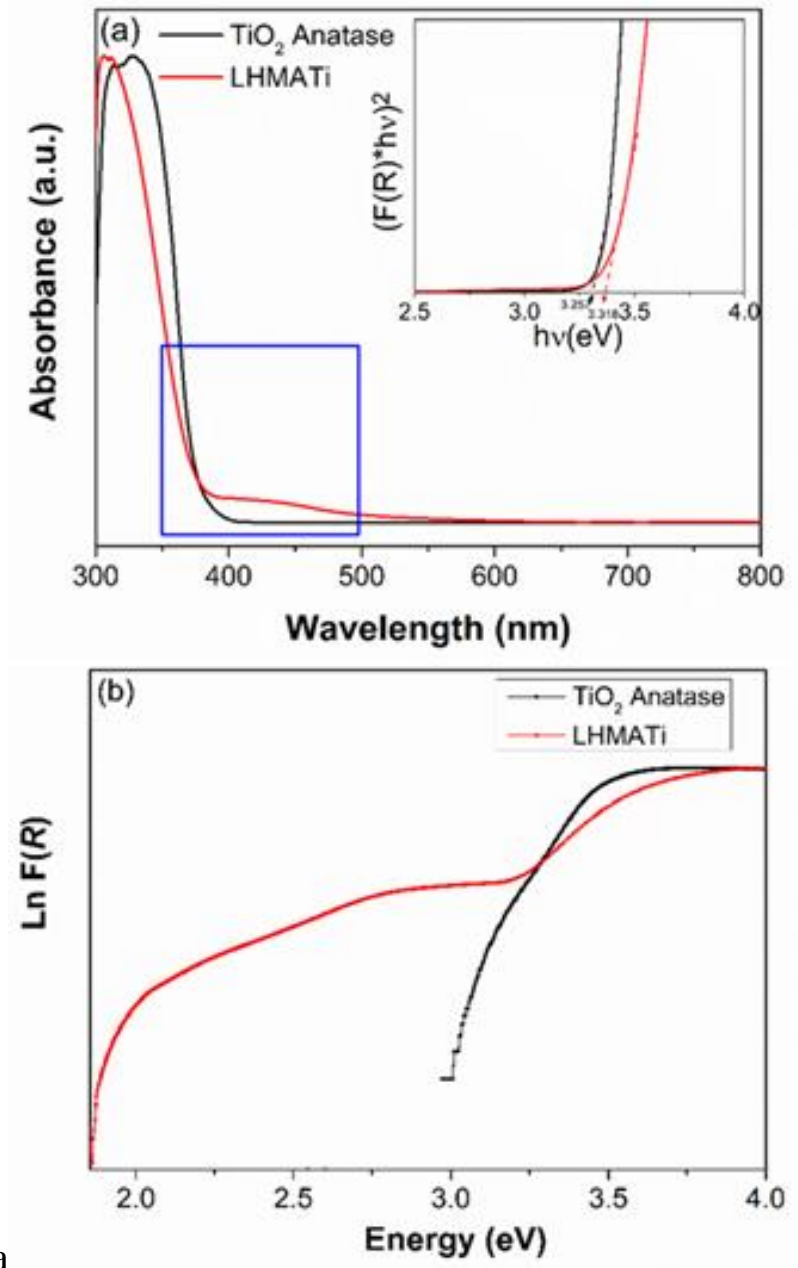

Figure S5. (a) UV-vis Diffuse Reflectance Spectra of $\mathrm{TiO}_{2}$ anatase and LHMATi sample 1, (Kubelka-Munck conversion). Inserted: Band gaps by Tauc equation. (b) The relation between absorption coefficient $\ln \mathrm{F}(\mathrm{R})$ and energy $h v$ for both $\mathrm{TiO}_{2}$ anatase and LHMATi sample 1. Data recorded on a Shimadzu UV-Vis Double Beam Spectrometer (Model 2450 PC) with integrating sphere assembly. Reference sample: $\mathrm{BaSO}_{4}$. 


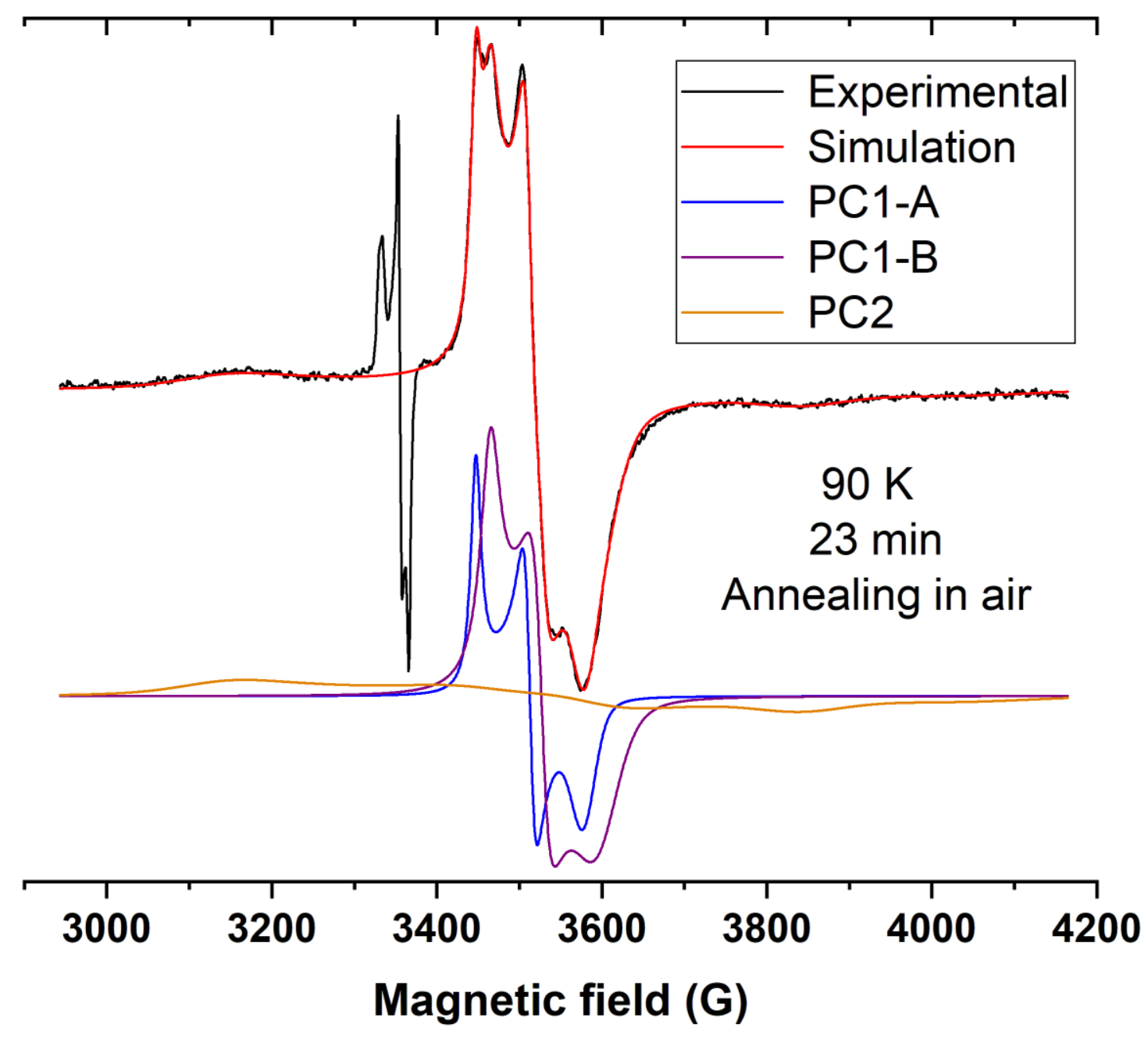

Figure S6. Simulation of the EPR spectrum shown in Figure 10 (Experiment 3) for annealing time of $23 \mathrm{~min}$ in air. Microwave frequency $=9.43248 \mathrm{GHz}$. 

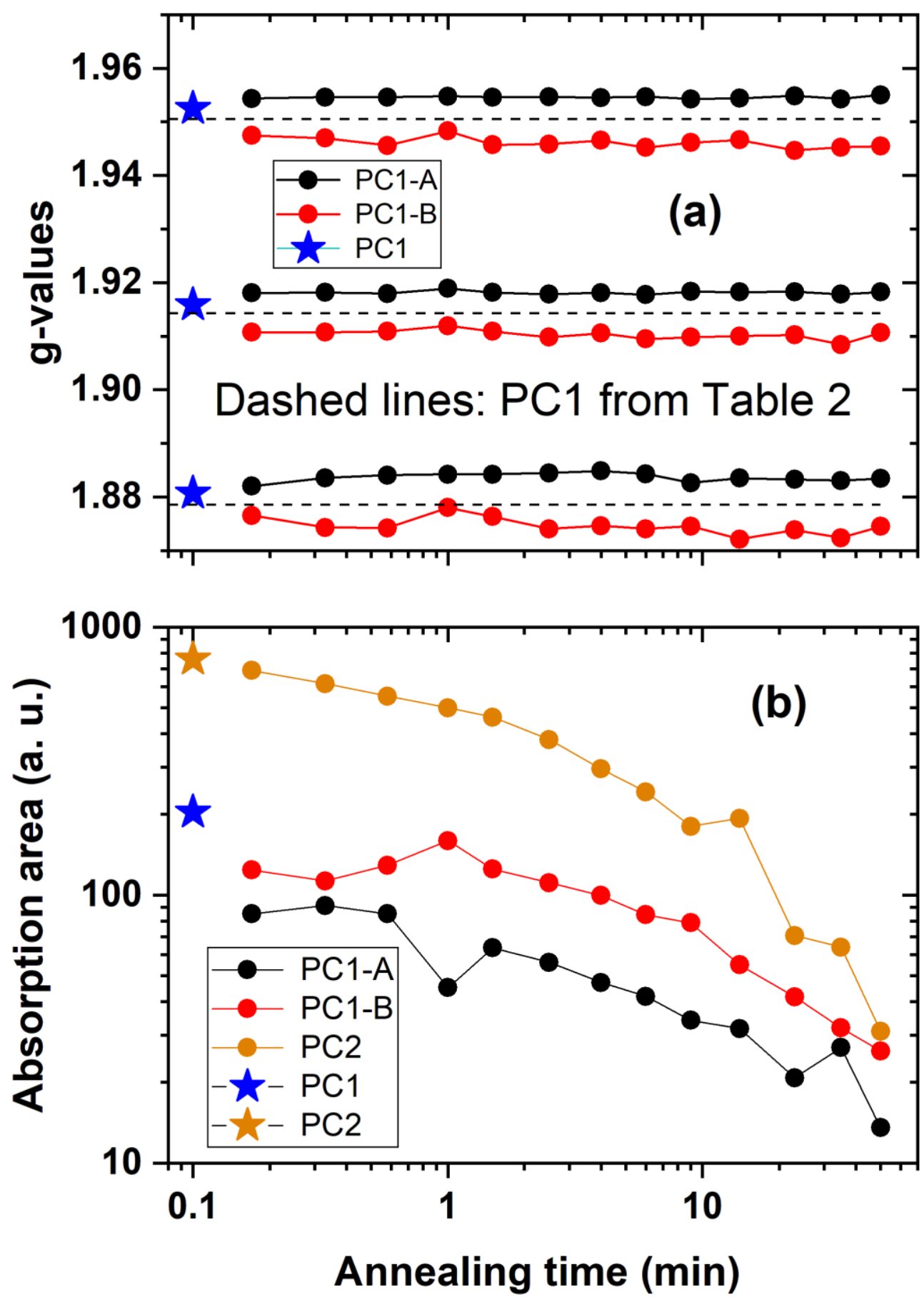

Figure S7. Simulation parameters of the PC1 and PC2 spectra of Figure 10 (Experiment 3). (a) Principal values of the g-tensor. (b) Integrated area of the absorption spectrum. The data points at $0.1 \mathrm{~min}$ (marked with stars) represent the values obtained from the spectrum acquired before annealing (top spectrum in Figure 10). Best fitting for the remaining spectra was achieved by the superposition of three components, PC2, PC1-A and PC1-B. 


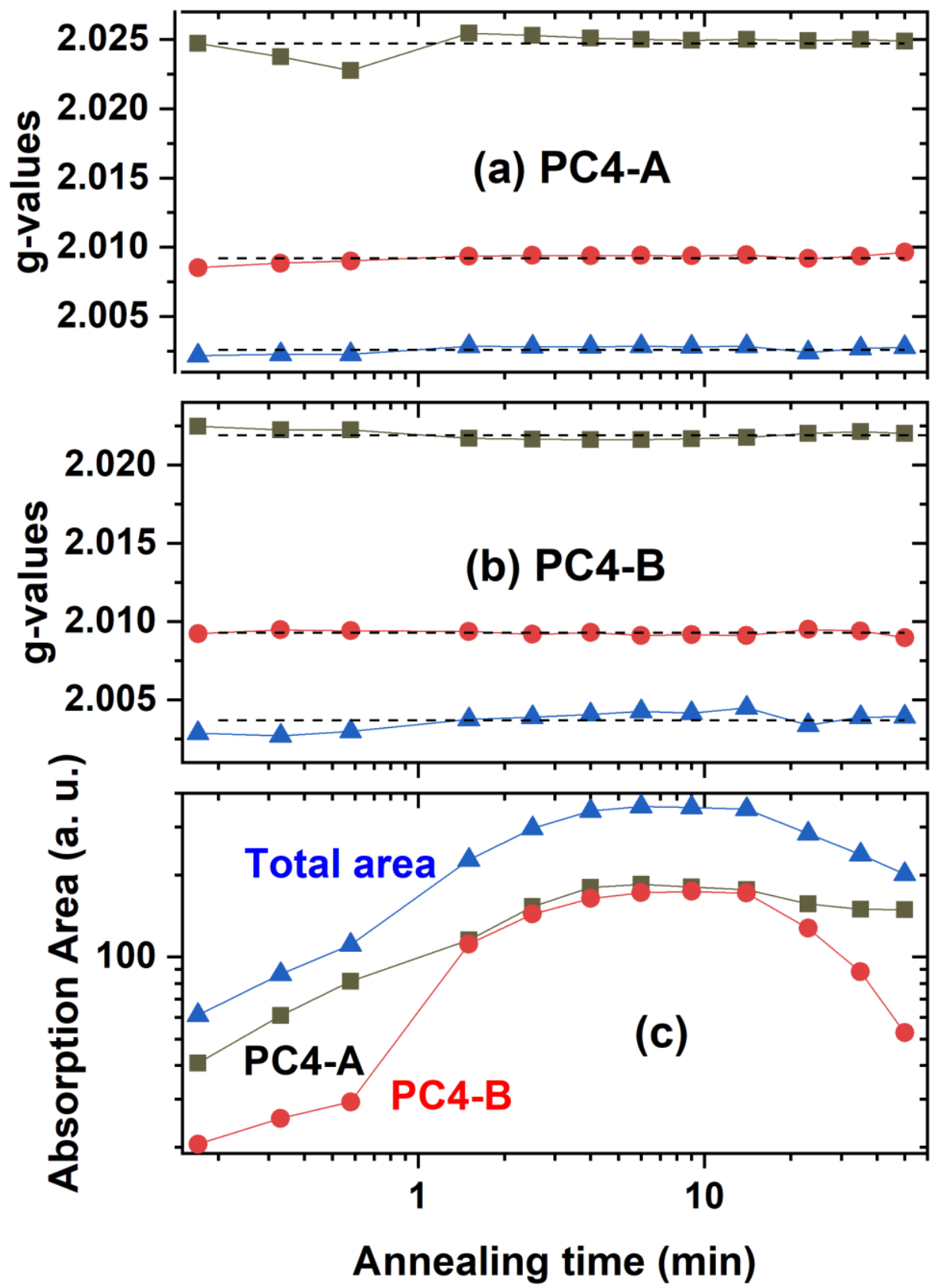

Figure S8. Simulation parameters of the PC4 spectra of Figure 10 (Experiment 3). (a,b) Principal values of the g-tensor. (c) Integrated area of the absorption spectrum. Total area is the sum of the areas of the two components. Dashed lines represent average values. Solid straight lines are a guide to the eyes. 


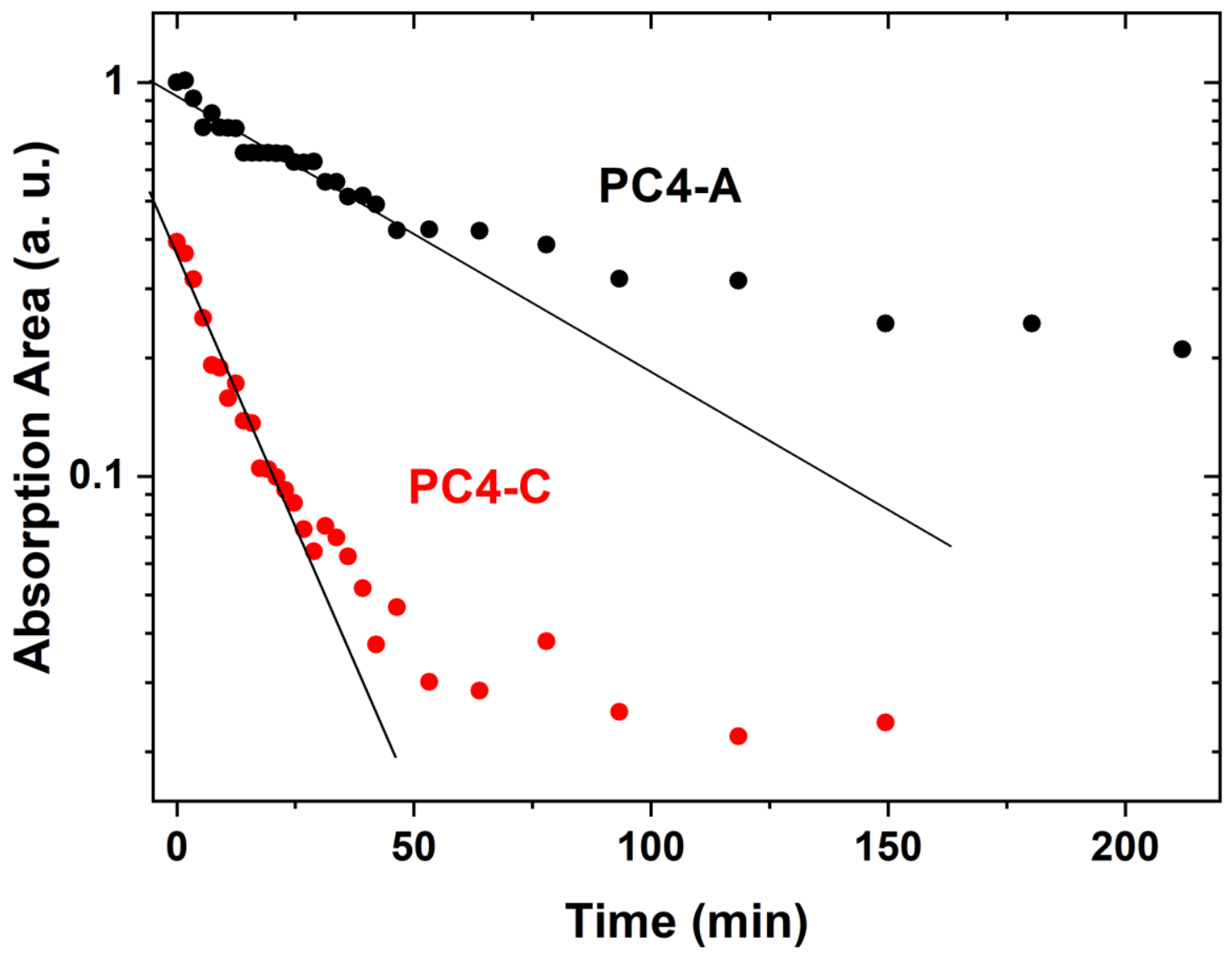

Figure S9: EPR relative intensity of paramagnetic centers PC4-A and PC4-C in experiment 4 as a function of time after 15 minutes of UV irradiation ( $365 \mathrm{~nm})$. Irradiation and measurement at room temperature. Straight lines are just guide to the eyes. Microwave frequency: $9.789 \mathrm{GHz}$. 

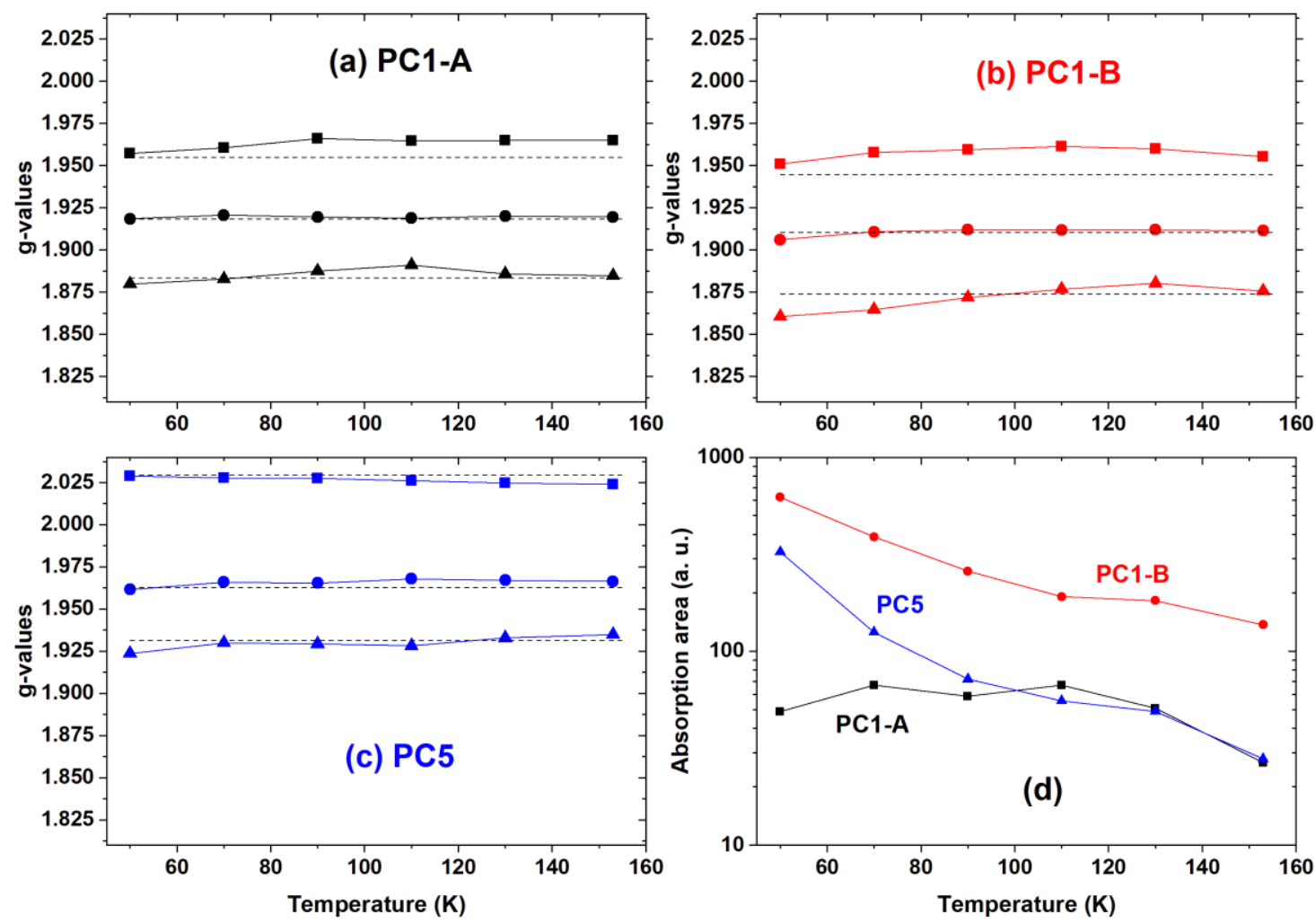

Figure S10. Principal values of the g-tensor (a-c) and absorption areas (d) calculated from the simulation of the PC1-A, PC1-B and PC5 spectra of Figure 14 (Experiment 5 in the range 50-153 K). Dashed lines denote the g-values listed in Table 2 for PC1-A, PC1-B and PC5.

\section{Additional Experimentation}

\section{Wavelength-dependent studies: Sample irradiated at $300 \mathrm{~K}$ in air and measured}

at $300 \mathrm{~K}$. Figure 12 of main text presented the EPR spectra recorded simultaneously with irradiation $(365 \mathrm{~nm})$ in air at $300 \mathrm{~K}$. The sample was kept inside the cavity-cryostat apparatus and the EPR signal was monitored simultaneously with irradiation as a function of time (procedure 1). Now, we present an analogous experiment, in which a new fresh portion of the same sample was irradiated at $420 \mathrm{~nm}$ and results are displayed in Figure S11. Before irradiation no EPR signal was observed, or the existing signal intensity was of the order or below spectrometer noise level; as indicated in the plot denoted by $0 \mathrm{~min}$. The observed spectra appear after few minutes of irradiation and increases in intensity reaching a stable level after about 20 minutes of irradiation. Three prominent spectral features can be identified and are marked by symbol *, that are coincident with the g- 
values of site PC4-A identified in the main text. In the case of the experiment described by Figure 12 of main text, the spectra had a much higher intensity than the ones shown here in Figure S11 and showed the additional appearance of a second set of g-parameters, there denoted by symbol $(\bullet)$. As discussed in the main article, that new species corresponds to the label PC4-C. However, PC4-C is not present in the sample irradiated at $420 \mathrm{~nm}$. Besides, no detectable signals appear when similar experiments were performed with the $530 \mathrm{~nm}$ (green) and $625 \mathrm{~nm}$ (red) light sources.

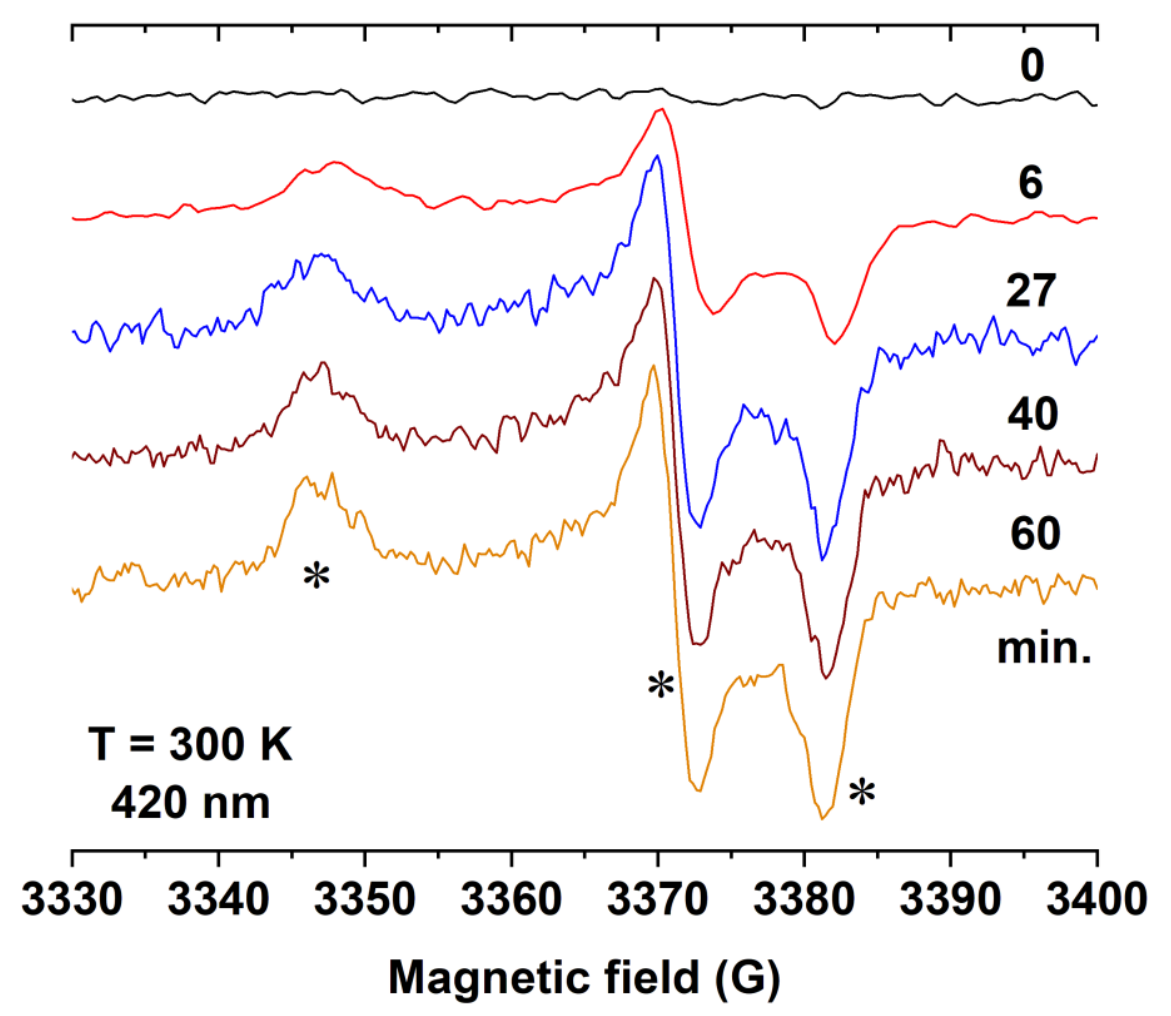

Figure S11. EPR signal measured simultaneously with irradiation $(420 \mathrm{~nm})$ in air at 300 K. Numbers on the right indicate irradiation time in minutes. Spectroscopic features corresponding to PC4-A component are marked by asterisks. Microwave frequency: 9.48 GHz.

2.Effect of irradiation time, irradiation temperature and temperature dependent EPR lineshape evolution. In this set of experiments, the non-evacuated sample was irradiated at $90 \mathrm{~K}$ with visible light (projector lamp) and simultaneously measured in steps up to a maximum exposure time of 60 minutes. Results displayed in Figure S12a indicate, 
as in the case of violet irradiation, that the spectra are dominated by the $\mathrm{Ti}^{3+}$ centers (PC1 species) and the superoxide radical (dominantly the PC4-A species). The signal intensities increase with increasing irradiation time.

Subsequently the lamp was turned off and EPR spectra were recorded at different temperatures as the sample was allowed to warm up from $90 \mathrm{~K}$ to room temperature. The spectra in Figure S12b shows that (1) superoxide is the only detectable species at room temperature and (2) for this irradiation condition, the dominant superoxide species is species PC4-A. Small amounts of PC4-C are present as well, showing little change as a function of temperature.

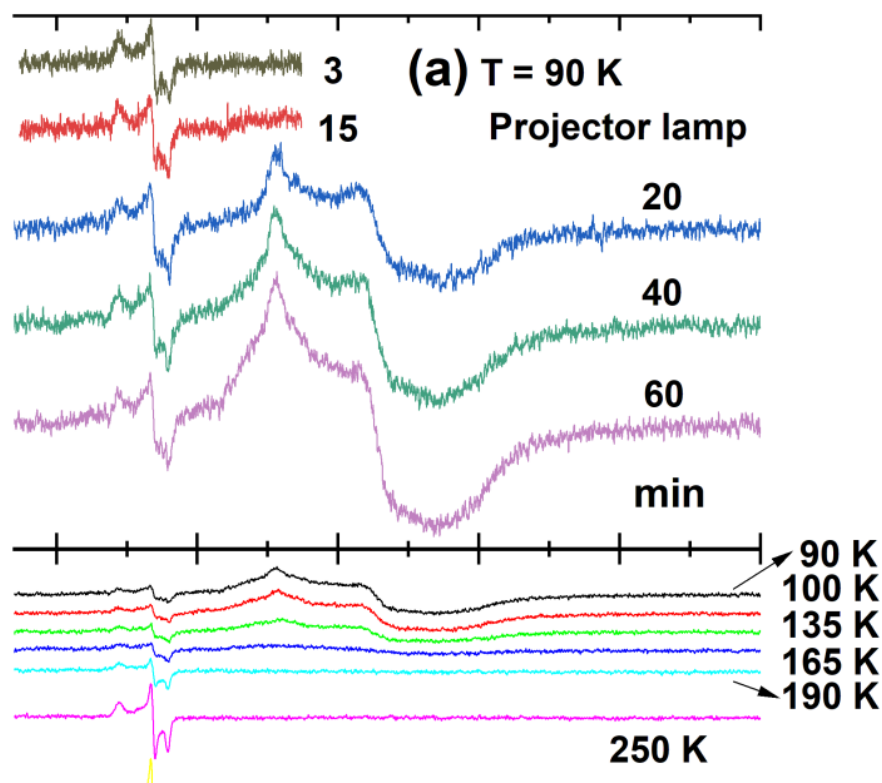

$260 \mathrm{~K}$

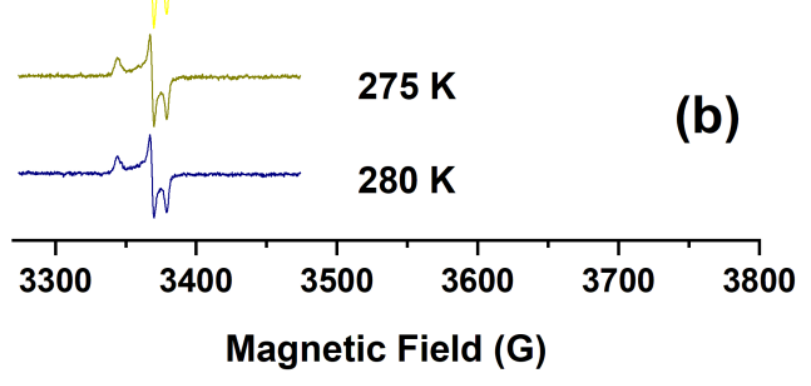

Figure S12. (a) EPR signal measured simultaneously with irradiation at $90 \mathrm{~K}$ (projector lamp). Numbers on the right indicate irradiation time in minutes. (b) After the sample being irradiated during 60 minutes the lamp is turned off and EPR spectra are taken as the temperature increases. Microwave frequency: $9.47 \mathrm{GHz}$. 
3.Temperature cycled annealing experiments conducted on samples irradiated at room temperature in the non-evacuated state. This study complements Figures 6,8 and 10 of the main text, the difference being that the $90 \mathrm{~K}$ intensity measurements of species PC4 monitored during the cycled room temperature annealing experiments were done on non-evacuated samples that were irradiated $(365 \mathrm{~nm})$ for $1 \mathrm{~h}$ at room temperature. The results are summarized in Figure S13. Overall, the results are principally similar to those obtained in Figure 10 (main text) on the samples irradiated in the evacuated state, and subjected to the subsequent $90 \mathrm{~K}$-> $300 \mathrm{~K}$ annealing cycles. Contrary to Figure 10, in the present experiments, a small amount of PC4-C species is present. Interestingly, a successful simulation could be achieved without considering the triplet species PC2, indicating that the amount of dimers produced in this experiment is can be neglected, as shown in Figure S14. In Figure S15 the integrated absorption areas of the EPR spectra of species PC1 and PC4 are plotted as a function of cumulative annealing time. The area of the PC1 component decreases monotonically while, for PC4-A and PC4-C, the area initially increases abruptly during the first 4 minutes of annealing and then decreases.

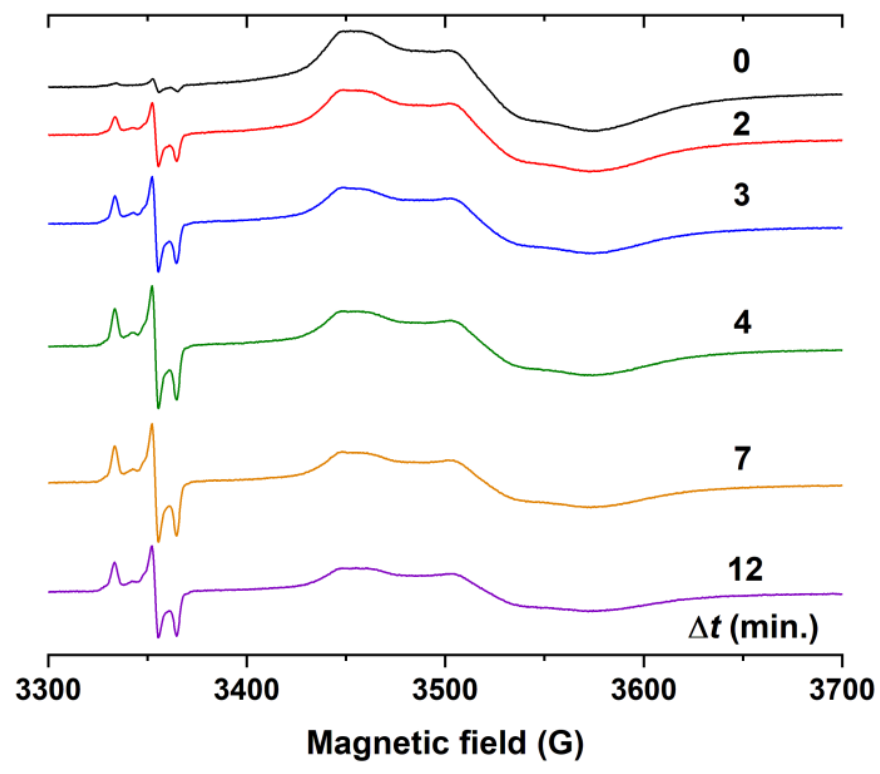

Figure S13. EPR spectra recorded at $90 \mathrm{~K}$ during various annealing/cooling cycles as described in the text. Microwave frequency: $9.43 \mathrm{GHz}$. 


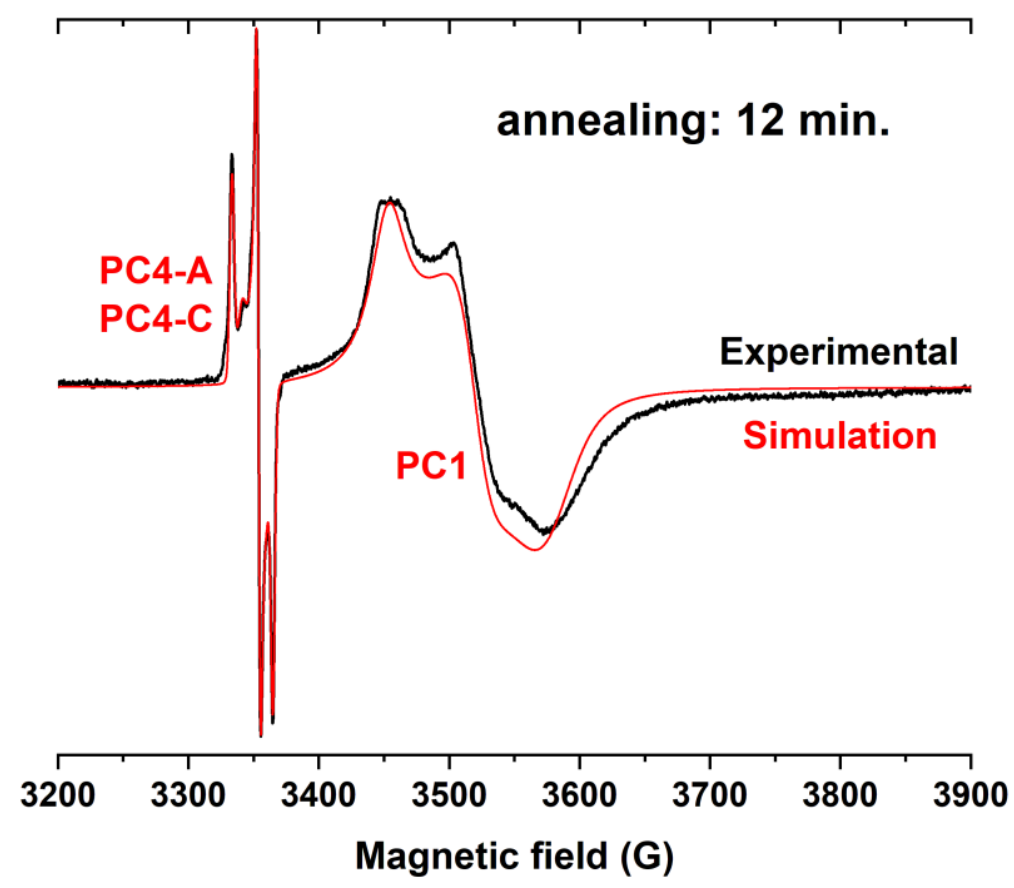

Figure S14. Simulation of the EPR spectrum shown in Figure S13 for annealing time of $12 \mathrm{~min}$. Microwave frequency $=9.4323 \mathrm{GHz}$.

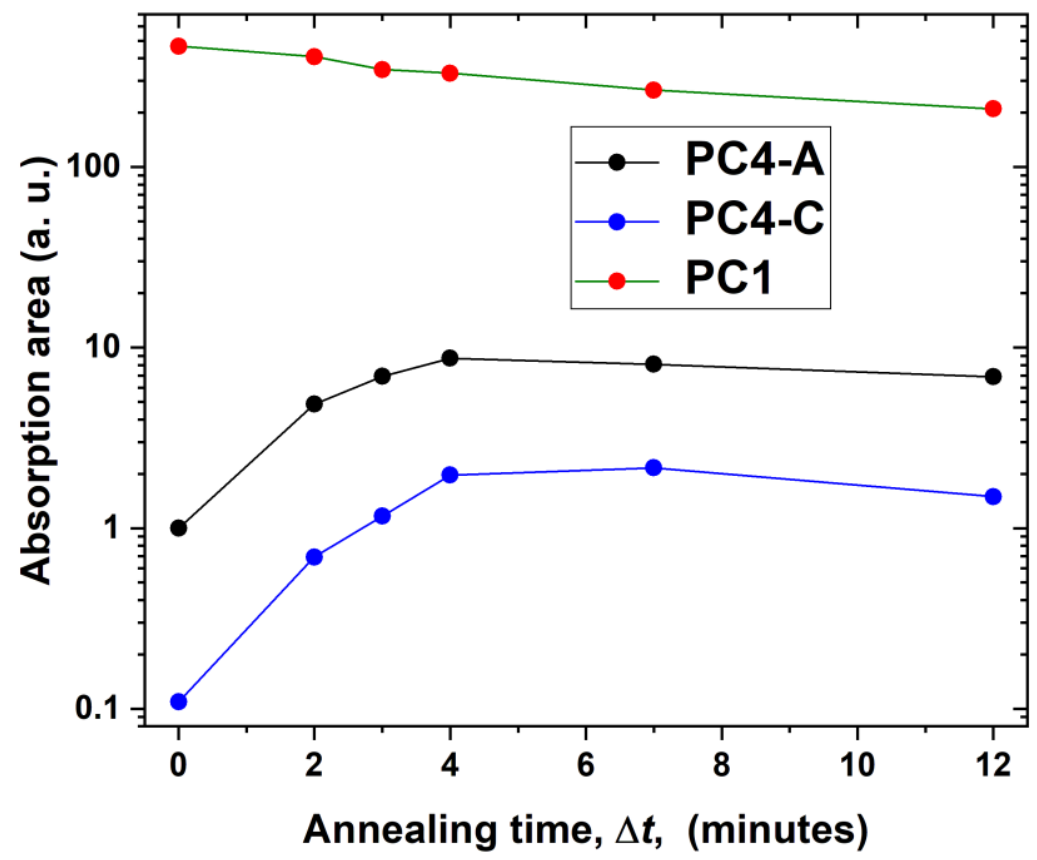

Figure S15: Integrated absorption areas of the EPR spectra shown in Figure S13, calculated from the simulated spectra of the paramagnetic centers PC1 and PC4. Microwave frequency: $9.43 \mathrm{GHz}$. 
4. Cycled annealing experiments involving annealing temperatures below $300 \mathrm{~K}$. To monitor the influence of annealing temperature, a cycled annealing experiment was conducted following the protocol shown in Figure S16. At the end of the cycled annealing study the signal was measured as a function of temperature, within the temperature range $170-260 \mathrm{~K}$. The results show that sample PC4-C is not being formed when the annealing protocol limits the annealing temperatures to $200 \mathrm{~K}$. This species is only observed at measurement temperatures above $200 \mathrm{~K}$

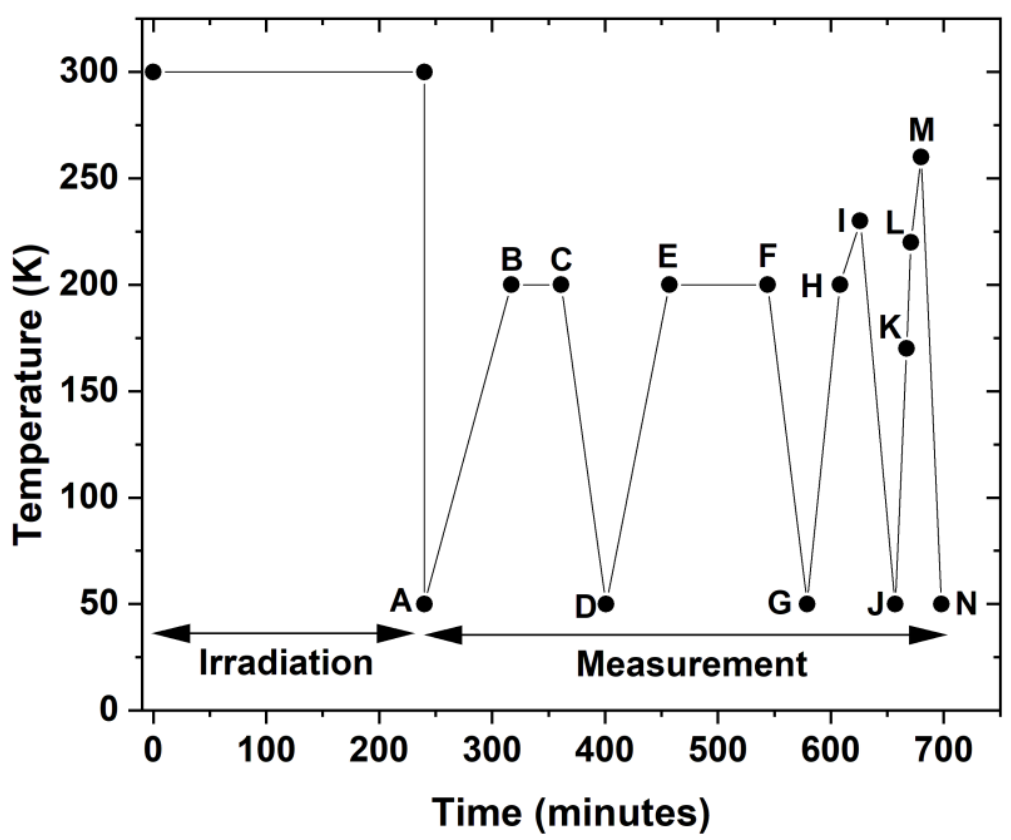

Figure S16. Annealing and measurement protocol conducted on a non-evacuated sample irradiated with $80 \mathrm{~mW} 365 \mathrm{~nm}$ UV source at $300 \mathrm{~K}$ for $240 \mathrm{~min}$, using annealing temperatures in the range $170-260 \mathrm{~K}$. 


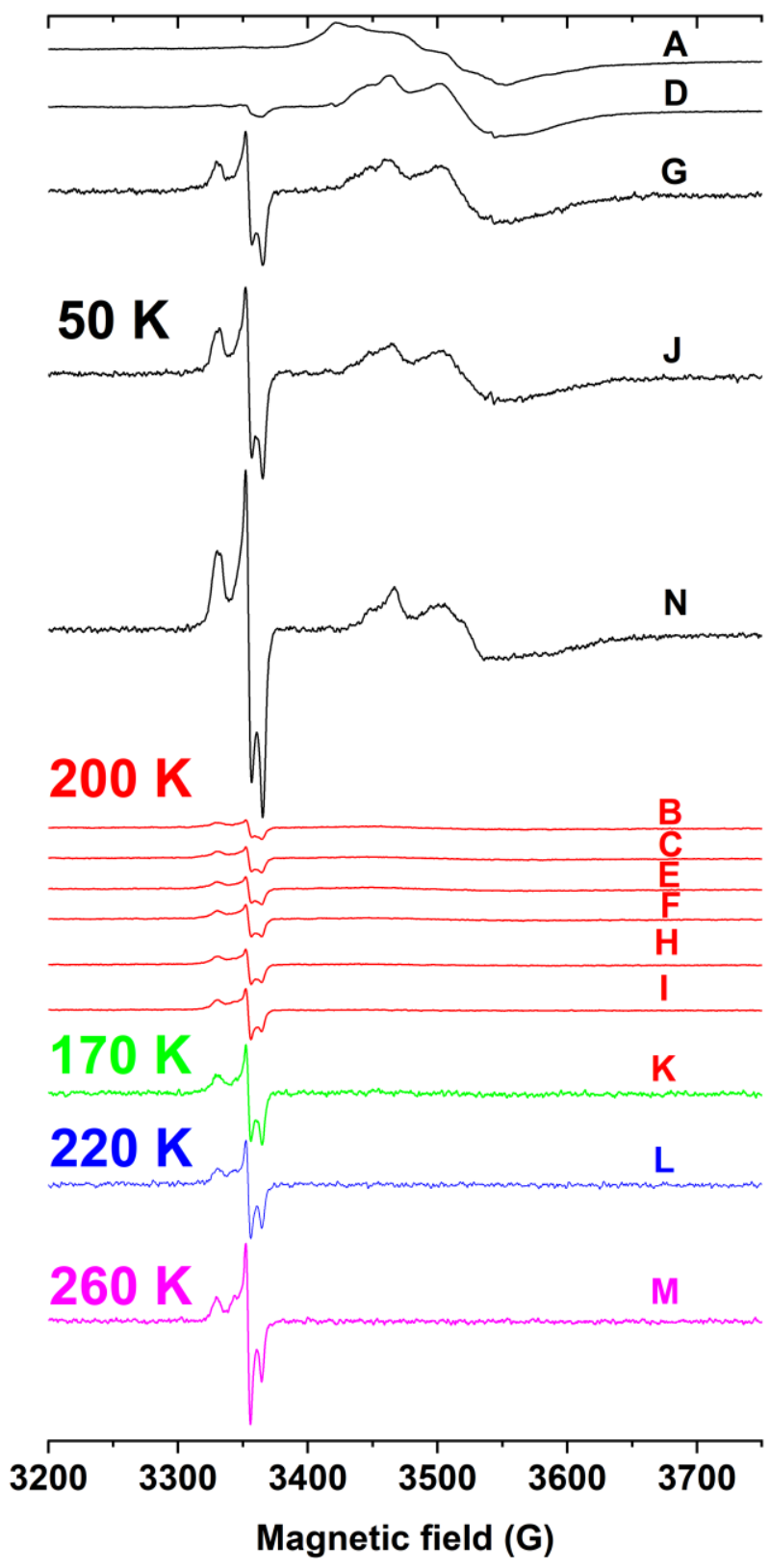

Figure S17. Results of the cycled annealing experiment conducted on a non-evacuated sample irradiated with $365 \mathrm{~nm}$ UV source at $300 \mathrm{~K}$ for $240 \mathrm{~min}$, following the protocol described in Figure S16. Microwave frequency: $9.43 \mathrm{GHz}$.

\section{Cycled annealing -variable temperature experiments}

In a final experiment, the heating/annealing cycle followed the temperature/time protocol listed in Figure S18, involving various heating/cooling cycles at variable temperatures. The results offer insights into the relationship between species PC4-A and PC4-C. 


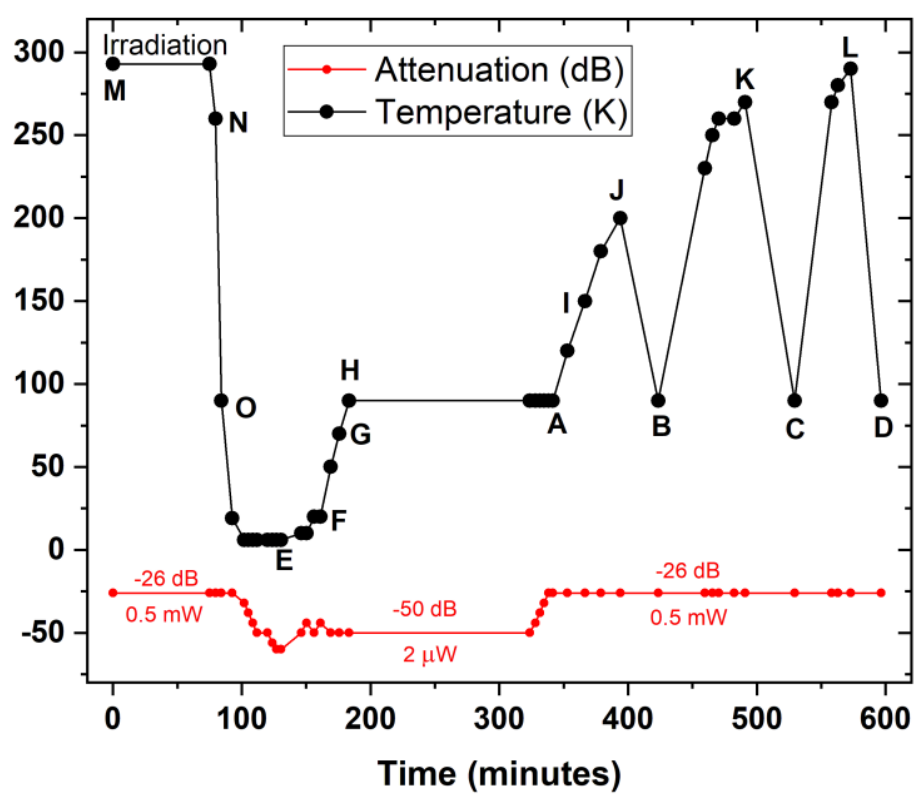

Figure S18: Time/temperature protocol of annealing/cooling cycles involving variable temperatures. Black data points show the temperature trajectory. Red data points show the microwave attenuation in $\mathrm{dB}$ used in each measurement. These adjustments were necessary to avoid saturation effects. Microwave frequency: $9.434 \mathrm{GHz}$.

We measured point $\mathrm{M}$ before irradiation (no signal). Then we irradiated the sample at 365 $\mathrm{nm}$, procedure 2, during 1 hour. After irradiation we moved the sample to inside the cryostat/cavity and started a fast cooling down. While cooling down, we measured at 260 $\mathrm{K}(\mathrm{N}), 90 \mathrm{~K}(\mathrm{O}), 10 \mathrm{~K}$ and $6 \mathrm{~K}$. The first two measurements were done "on-passage" as the temperature was decreasing; therefore, the temperature reading may not be exact.

The first four measurements are plotted in Figure S19. Note that, while the spectrum reveals the same features as the $90 \mathrm{~K}$ spectrum in Figure 10 (top trace), the PC4/PC1 intensity ratio is much higher in the present case. Saturation studies conducted at $6 \mathrm{~K}$ indicate that PC1 saturates very little, whereas the PC4 species are characterized by very long spin-lattice relaxation times, requiring microwave power attenuation of $50 \mathrm{~dB}$ for recording undistorted spectra. 


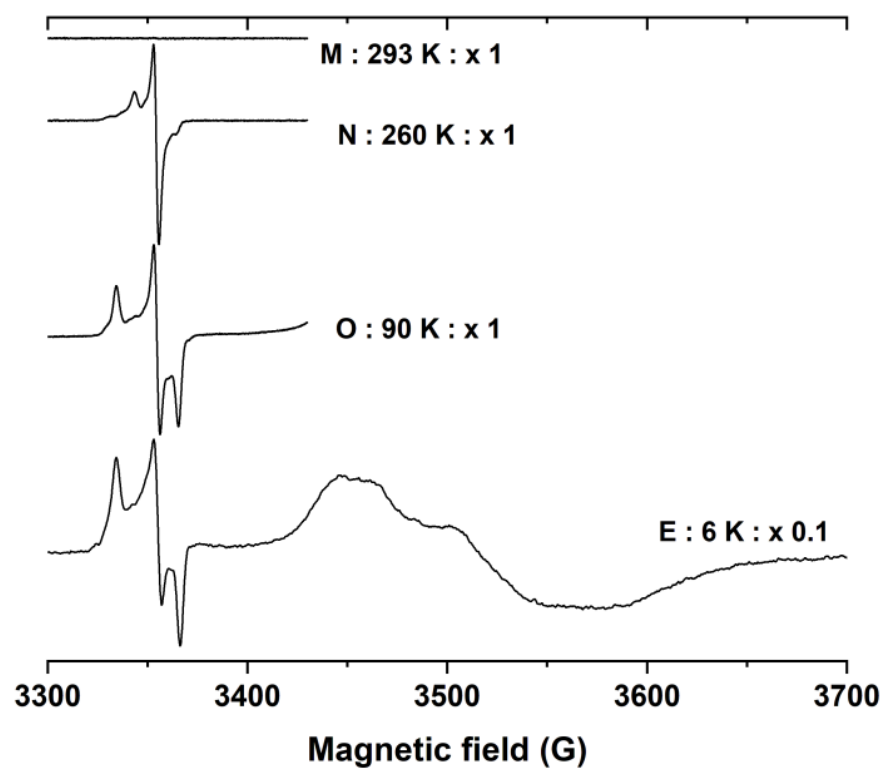

Figure S19: EPR spectra recorded during cooldown. Microwave frequency: $9.434 \mathrm{GHz}$.

Continuing the experiment, we increased the temperature monitoring the EPR signal at intervals. Some of the data is shown in Figure S20. As we increased the temperature, we did some jumps back to $90 \mathrm{~K}$ to check the centers (at points A-D) and to monitor the progress of the radical transfer reaction. The result is summarized in Figures S20, S21 and $\mathrm{S} 22$.

The low temperature lineshapes, below $90 \mathrm{~K}(\mathrm{E}, \mathrm{F}, \mathrm{G}, \mathrm{H})$, could be well fitted by a combination of PC4-A, PC4-B and PC4-C species with g-tensor values consistent with those determined before (Table 2). Likewise, the spectra A,B,C,D obtained by repeated cycling down to $90 \mathrm{~K}$ can be fitted with this set of parameters. Overall, the results confirm the expected relationship between PC4-A and PC4-C. The higher the temperature of measurement, the lower the PC4-A/PC4-C ratio. This result suggests that with increasing temperature PC4-A transform into PC4-C species. Thus PC4-C may represent the same chemical species, i.e. the superoxide radical, in a different anisotropic molecular dynamical state. Further support for this hypothesis comes from the observation that the isotropic g-values of both PC4-A and PC4-C, are exactly identical. 


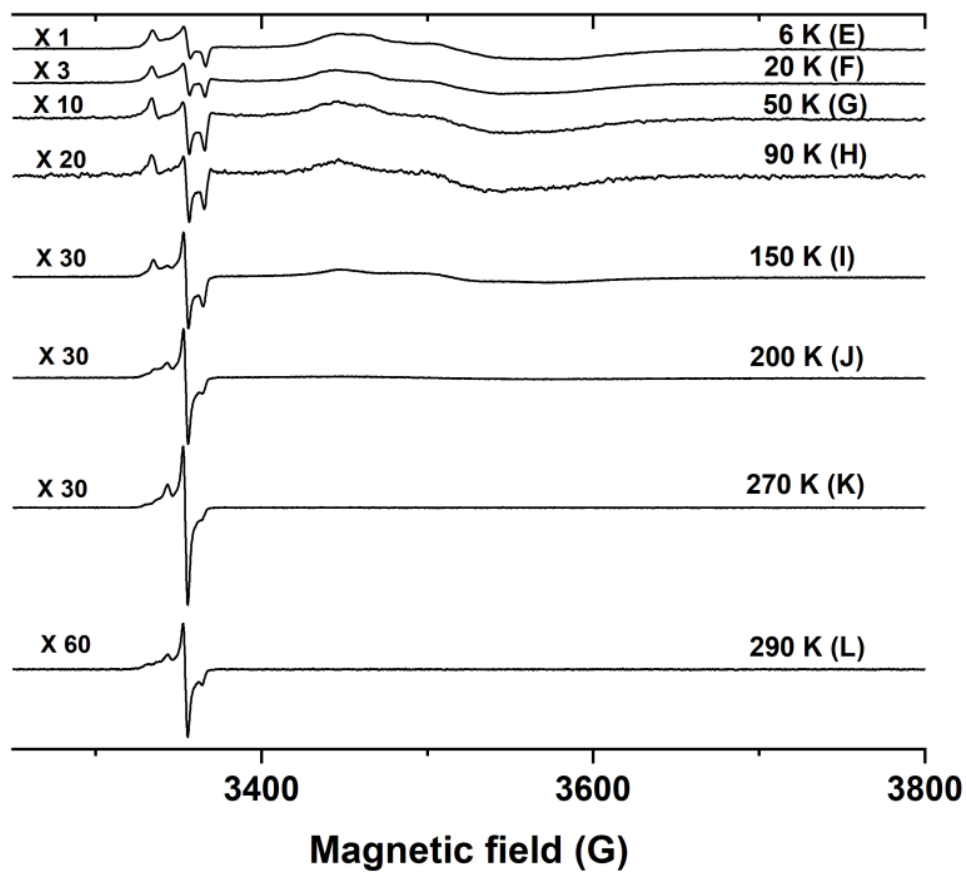

Figure S20: EPR data taken along the thermal trajectory indicated in Figure S18 Microwave frequency: $9.434 \mathrm{GHz}$.

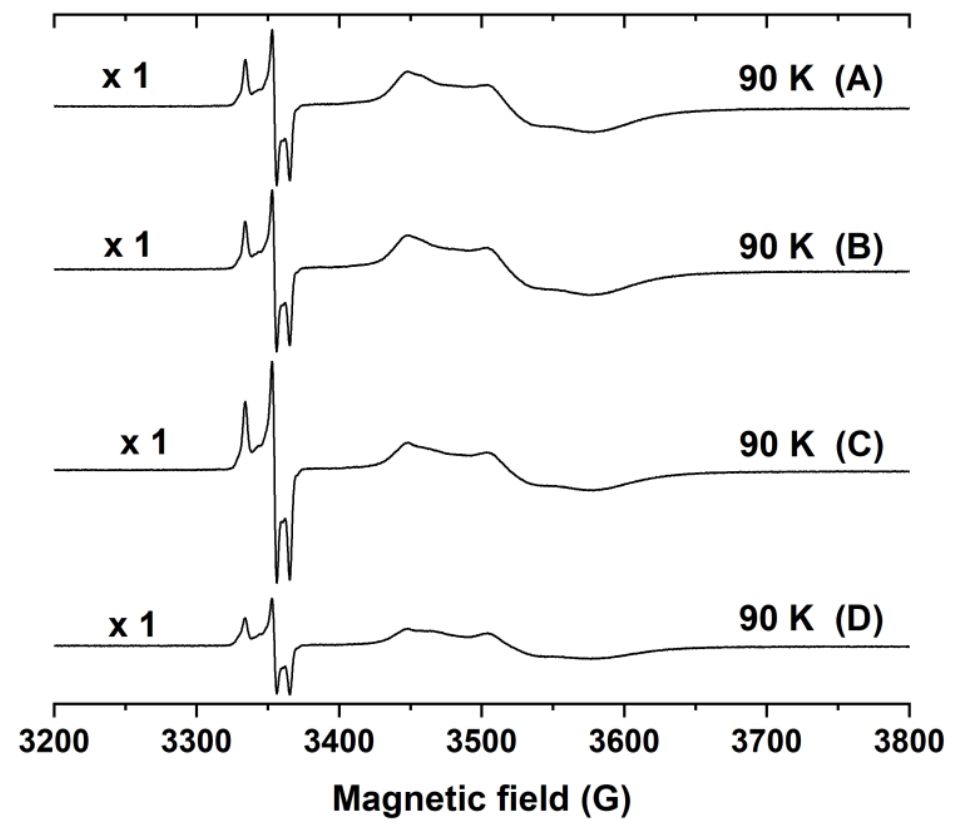

Figure S21: EPR data taken along the thermal trajectory indicated in Figure S18. The reproducibility of the $90 \mathrm{~K}$ spectra indicate that the spectral changes are reversible. This result suggests that the lineshape changes are due to temperature-dependent shifting chemical equilibria or kinetic processes. Microwave frequency: $9.434 \mathrm{GHz}$. 


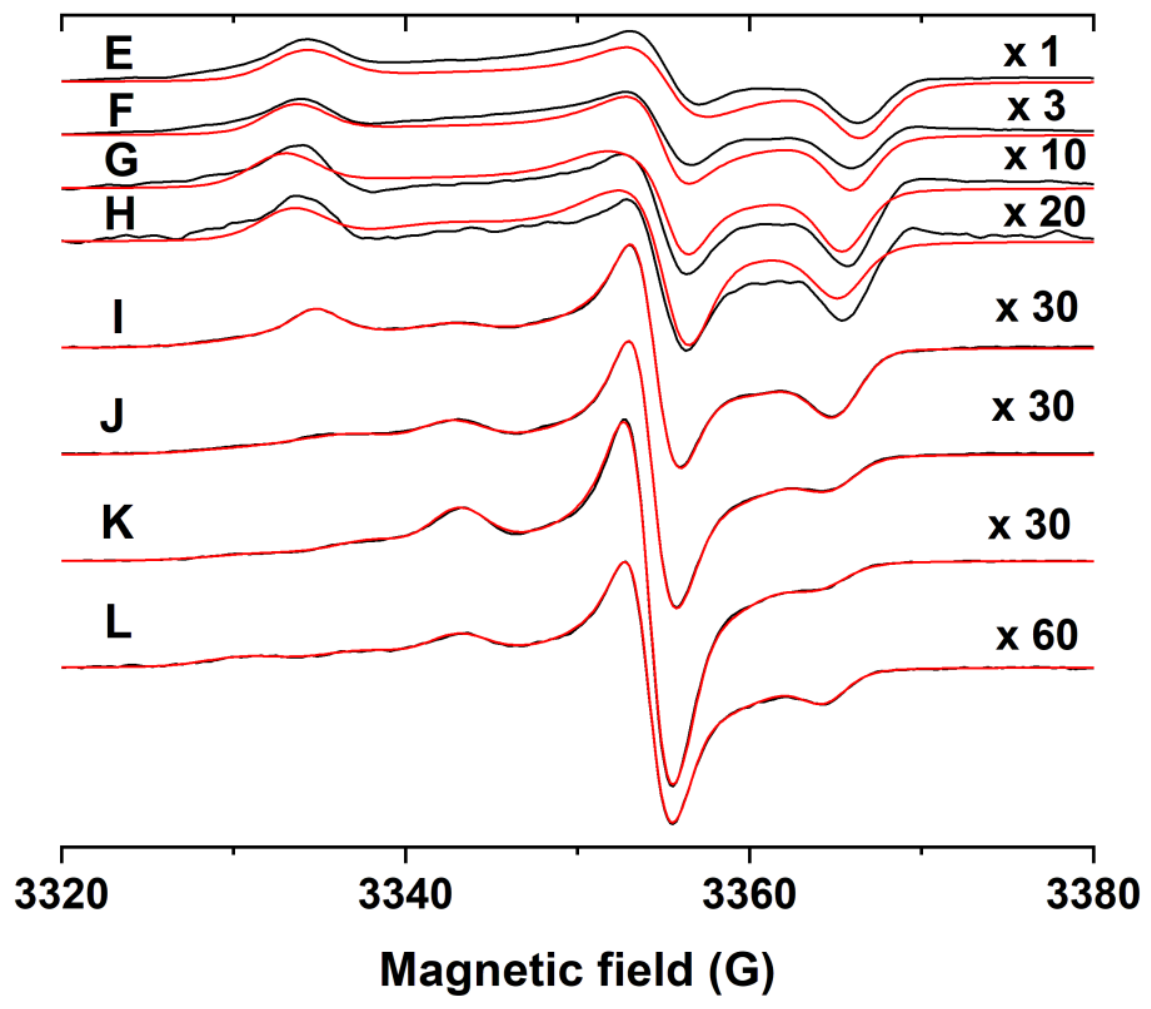

Figure S22: Fit of the $\mathrm{g}>2$ portion of the spectra of the three species PC4A-C. Black: experimental. Red: simulation. 